American Journal of Environmental Sciences 6 (2): 159-163, 2010

ISSN 1553-345X

(C) 2010 Science Publications

\title{
Evaluation of Aerosol Particle Size at High Polluted Region in the World Using Direct Solar Radiation Measurements: Helwan as a Case Region
}

\author{
U. Ali Rahoma \\ Department of Medical Radiology, Faculty of Medical Technology, \\ Omer El-Mukhtar University, Tobruk, Libya
}

\begin{abstract}
Problem statement: Particle counting and sizing of atmospheric aerosols by electro-optical methods are complex and absolute interpretation of measurements is difficult, so, the scattered light varies in a complicated manner with the system of optics as well as with the size and physical characteristics of particles. Approach: The estimation of the air aerosol number concentration variation was carried out in this study making use of data obtained from the Helwan, Egypt. The aerosol number concentration was determined indirectly, making use of the intensity of light scattered by particles. The scattered light intensity was proportional to the average number concentration of the aerosols. Results: The results were presented from desert zone from using Eppley direct solar irradiance measurements as a base on 10 years of data collection (1991-2000). Conclusion: The differences among the region were characterized mainly by their different climate change taken in consideration in the spectral region 250-900 $\mathrm{nm}$. Most of the particles are greater than $10 \mu \mathrm{m}$ in aerodynamic diameter and $60-80 \%$ of particles was $5-10 \mu \mathrm{m}$ and was trapped in the nasopharyngeal region. This showed an idealized size distribution of particulate matter in ambient air and measurement techniques to cover specific fractions. The columnar volume radius distributions of aerosol 3-6 $\mu \mathrm{m}$ showed the aerosol optical depth is less than 0.31 for $\lambda=500-630 \mathrm{~nm}$. The behavior in a more turbidity, when the aerosol optical depth is about 0.25 for $630-695 \mathrm{~nm}$, gives approximately fixed volume radius distributions of aerosols between 5-10 $\mu \mathrm{m}$. The small size fraction of aerosols, measured as $\mathrm{PM}_{10}$ and $\mathrm{PM}_{2.5}$, rather than the larger particles, was considered to be responsible for most of the health effects.
\end{abstract}

Key words: Aerosol optical depth-particle size-direct solar irradiance-spectral region-scattered light

\section{INTRODUCTION}

The aerosol number concentration is determined indirectly, making use of the intensity of light scattered by particles. The scattered light intensity is proportional to the average number concentration of the aerosols. The advantages of modeling aerosols with their lowerorder moments, rather than the full size distribution itself has been discussed by McGraw et al. (1995; 1998); McGraw (1997) and Barrett and Webb (1998) and elsewhere. Aerosol dynamics via the Quadrature Method of Moments of McGraw (1997) has now been incorporated in the Eulerian chemical transport and transformation model of Benkovitz et al. (1994) and the researcher will report on such simulations in the near future. The real atmosphere contains different particles - interacting with solar radiation, such as gas molecules, aerosol particles of different size, shape and chemical composition and cloud droplets (Ferm, 1979). Therefore, we are interested in the interaction not with the separate particles but with a total combination of them (Melnikova and Vasilyev, 2005). Aerosols are defined as suspensions of liquid or solid particles in the air, excluding cloud droplets and precipitation. The mean radii of aerosol particles range from about 4-10 microns. William and Barbara (2001) observed that wind speed, mixing between air layers, precipitation and atmospheric chemistry determine whether pollutants that will remain in the locality, where they are produced or go elsewhere. Utah (1995) noted that there is a gradation in size of aerosols entrained in the North East trade winds blowing across West Africa during the harmattan months as the aerosols are transported further away from the Saharan source. Chiemeka (2006) noted that aerosols are important because they are able to alter climate in two ways. Firstly, they scatter and absorb radiation in the atmosphere and secondly they change the microphysical structure and possibly the life time and extent of clouds within the atmosphere. Also Chiemeka et al. (2007a; 2007b) observed that aerosols are characterized by low relative humidity and 
degradation of visibility, attenuation of radio signals and depletion of solar radiation. The number of ultrafine particles (diameter $0.01-0.1 \mu \mathrm{m}$ ) as well as the mass of a fraction of fine particles with 0.1-0.5 $\mu \mathrm{m}$ aerodynamic diameter was found to be associated with an indicator of pulmonary function in a study conducted in Germany (Peters et al., 1997).

Data base: The measurements were made under clear sky conditions (cloudless) (Eppley, NIP) and four large pass band flat filters of the types OG 530, 630, 695 and RG870. A system was installed on the roof of the new building of the National Research Institute of Astronomy and Geophysics (NRIAG) in Helwan (latitude $29^{\circ} 52^{\prime} \mathrm{N}$ and longitude $31^{\circ} 20^{\prime} \mathrm{E}$ ). Helwan is located about $30 \mathrm{~km}$ south of Cairo in a desert area. Data collections are presented from desert zone from using Eppley direct solar irradiance measurements after 10 years of continuous measurements of trends and variations in aerosol optical depths AOD (1991-2000). The significant differences among the parameters of the different methods related to the inversion methods of the AOD spectra also dealt. The general climate of Helwan dry, hot and desert type, the Nile and adjacent narrow cultivation belts have very limited local modifications on the climate. A strong pollution produced by the establishment of various industries in the neighborhood is also considered. The prevailing wind direction is from the north and northeast, which represents about $50 \%$ of the total directions. This leads to an important result, that is, cement factories contribute approximately $50 \%$ to the air pollution of the site. The major source of the wind directions is from the $\mathrm{N}$ and $\mathrm{N}-\mathrm{E}$, which represents about $50 \%$ of the total direction. The national cement factory and iron steel factories represent about $40 \%(\mathrm{~N}-\mathrm{W}, \mathrm{S}, \mathrm{W}, \mathrm{S}-\mathrm{W}$ directions) of the total pollution at Helwan region. Therefore, all the factors have been considered for this study.

\section{METERIALS AND MATHODS}

Method used to estimate particle size: The real atmosphere contains different particles-interacting with solar radiation such as gas molecules, aerosol particles of different size, shape and chemical composition and cloud droplets. It is possible to attribute the special characteristics of the interaction between the atmosphere and radiation to an elementary volume (formally infinitesimal) of this continuous medium. Scrutinizing the elementary volume of this continuous medium $\mathrm{dV}=\mathrm{dS} \mathrm{dl}$ (Fig. 1), on which the parallel flux of solar radiation $F_{0}$ incomes normally to the side $\mathrm{dS}$.
The scattered light has the same frequency (that is, the same wavelength) as the incident light. Independent scattering or independent particles are considered (that is, the scattering by well defined separate particles). Let $\mathrm{I}_{0}$ be the intensity of the incident light, $\mathrm{I}_{\mathrm{sc}}$ the intensity of the scattered light at a point located at a large distance $\mathrm{R}$ from the particle to the photo detector and $\mathrm{k}$ the wave number defined by:

$$
\mathrm{k}=2 \pi / \lambda
$$

where, $\lambda$ is the wavelength in the surrounding medium. Since $I_{s c}$ must be proportional to $I_{0}$ and $R^{-2}$ we may write:

$\mathrm{I}_{\mathrm{sc}}=\mathrm{I}_{\mathrm{o}} \frac{\mathrm{F}(\theta, \varphi)}{\mathrm{K}^{2} \mathrm{R}^{2}}$

For spherical particles, there is no phase dependence $(\mathrm{j}=0)$ hence we may write Eq. 1 as:

$I_{s c}=I_{o} \frac{F(\theta)}{K^{2} R^{2}}$

Here:

$\mathrm{F}(\theta)=$ A dimension less function

$\left(\mathrm{F} / \mathrm{K}^{2}\right)=$ An area of the direction but not of $\mathrm{R}$

It also depends on the orientation of the particle with respect to the incident wave and on the state of polarization of the incident wave (Van Dehulst, 1957). Then we may write:

$\mathrm{R}=1+\mathrm{u}$

Where:

$1=$ The radius of the cylindrical tube

$\mathrm{u}=$ The distance from outside of the cylindrical scattering medium to the detector surface

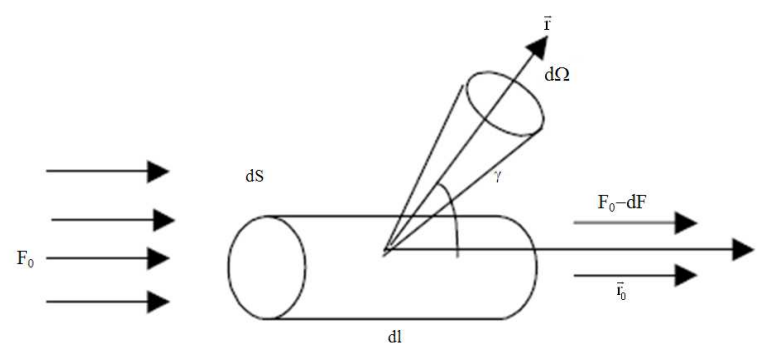

Fig. 1: Interaction between radiation and elementary volume of the scattering medium Melnikova and Vasilyev (2005) 
Therefore:

$$
I_{s c}=\frac{N a^{2} F(\theta) I_{o}}{X^{2} U(2 \lambda+\mu)}
$$

where, $\mathrm{a}$ is the radius of the particle, Where $\mathrm{x}$ is the size parameter $x=2 \pi / \lambda$. If the particle interacted with radiation according to geometric optics laws and was a non-transparent one, attenuated energy would correspond to energy incoming to the projection of the particle on the plane perpendicular to the direction of incoming radiation $r_{0}$ and its area is simply called a cross-section:

$$
I_{s c}=\frac{N a^{2} F(\theta) I_{o}}{X^{2} u(2 \lambda+u)}
$$

where, $x=2 \pi / \lambda$. The relation of the volume size distribution reveals that $v(\mathrm{r})=\mathrm{d} v / \mathrm{d} \log \mathrm{r}$ of the subtropical air mass exceeds that of the arctic air mass in the size range $0.06 \mu \mathrm{m} \leq \mathrm{r} \leq 8 \mu \mathrm{m}$ with a pronounced maximum covering the size range $0.06 \mu \mathrm{m} \leq \mathrm{r} \leq 0.5 \mu \mathrm{m}$ (Weller et al., 2000).

\section{RESULTS}

The distribution of frequency of occurrence for aerosol optical depth has a peak value at 0.2-0.4 and the yearly average is approximately 0.48 . There are several secondary peaks in March and April, associated with either small or large (relative to the average conditions) Angstrom exponents, less than 0.05 in January and $\sim 0.3$ in June. The maximums are associated with small Angstrom exponents (less than 0.3) which indicates that dust is the main contributor to the optical thickness (Fig. 2a-d).

The behavior of the mode strength along the period of campaign as shown in Fig. 3, where values are plotted for each mode radius: The first value is the average over the monitoring and the second value is the average over the afternoon data.

Result (Fig. 4) showed that for the $6 \mu \mathrm{m}$ mode a bell shaped behavior is clearly recognized with a maximum located in the middle of the day. The analysis of the strengths of the maxima and minima of $v(r)$, are also recognizable in the behavior of the aerosol optical depth. In Fig. 5, for each day of measurement, the values of $\tau_{\mathrm{A}}$ at $\lambda=500$ and $695 \mathrm{~nm}$, average over the morning and the afternoon, respectively, are shown; in particular for $\lambda=$ 500, during the first major event 25 May, 1999 the aerosol. Optical depth reaches the maximum value of 0.86 , followed by a sudden decrease in the afternoon of 25 may (Fig. 6) when it reaches the minimum value of 0.13 . The second major event 1-3 June shows almost the same maximum value of the first one, while the other two minor events 4-9 and 22-25 May shows a maximum value of 0.38 and 0.29 , respectively.

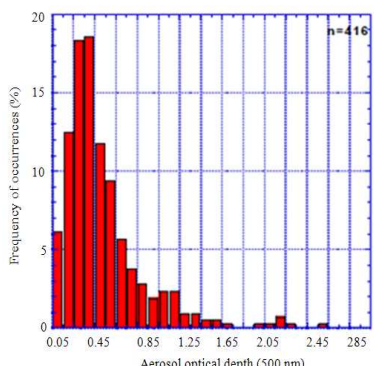

(a)

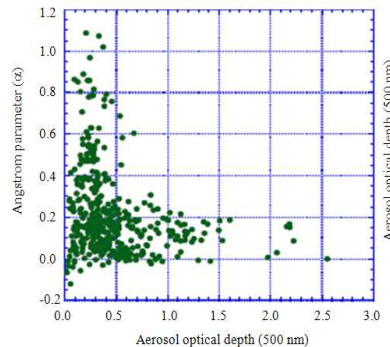

(c) (b)

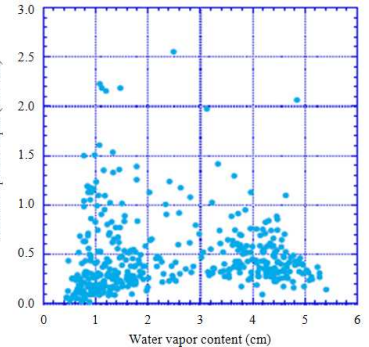

(d)

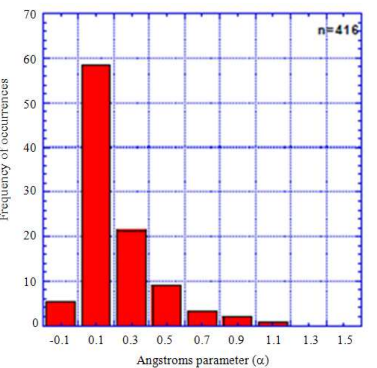

Fig. 2 a-d: The monthly means of $\tau a_{500}$ variability in the length of the suspended particle burning season in 1991 than in 2000

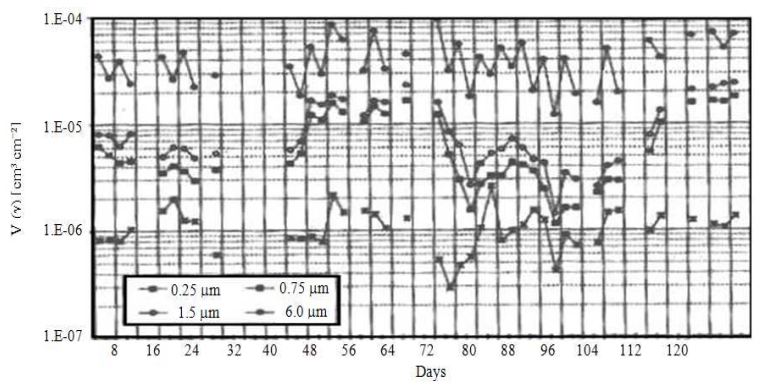

Fig. 3: Behavior of the strength of the four modes radii during the estimation Particle size at Helwan from the collecting days first of spring during Khamasien depression

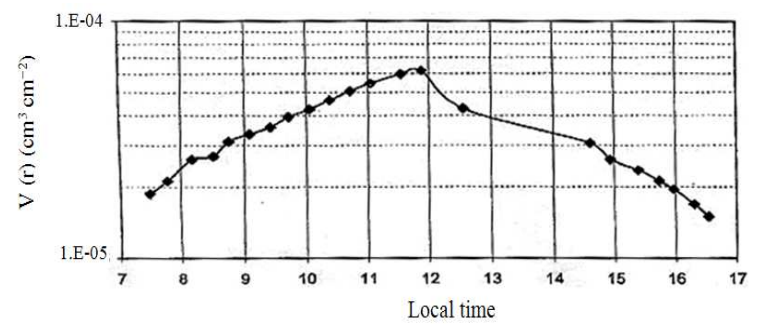

Fig. 4: Typical diurnal behavior of the strength of the $6 \mu \mathrm{m}$ mode radii August, 1999 


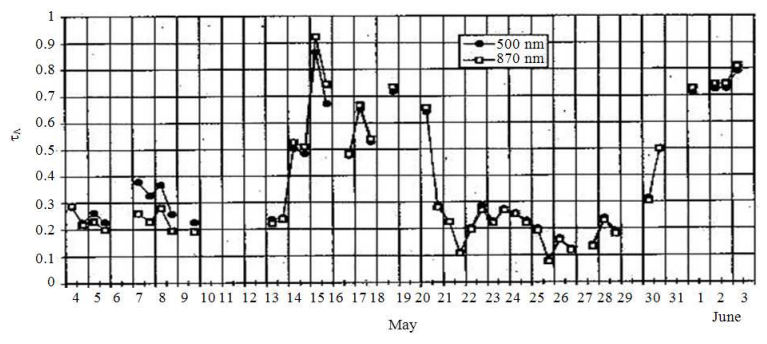

Fig. 5: Behavior of the aerosol optical depth at $\lambda=500$ and $870 \mathrm{~nm}$, during the campaign. For each day and for each wavelength two values are plotted, average over the morning and over the afternoon, respectively

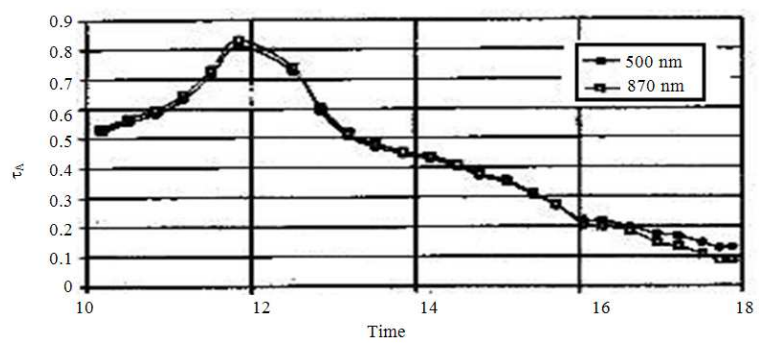

Fig. 6: Behavior of the aerosol optical depth on 25 May, 1999

\section{DISCUSSION}

The higher values of the Angstrom parameter in August and October. Correspond to the presence of the change from the Mediterranean depressions of the winter to the Khamasien depression of spring causes a rather sudden rise in temperature. The sudden change from the summer time regime to the winter one, often occurring in late September, causes a very drastic alteration in mean monthly temperature. The highest values of the maximum temperature occur in July and August. The maximum temperature reached $40^{\circ} \mathrm{C}$ in most places, the lowest temperature are encountered in winter in the heart of the western desert, between 25 and $29^{\circ} \mathrm{N}$ and west of $31^{\circ} \mathrm{E}$. It is more obvious in December when $\alpha$ reaches it's maximum (0.45). August and September are relatively clean months due to the scavenging of the atmosphere by precipitation. From March through June, the very low Angstrom exponent confirms the presence of dust. From the analysis of this behavior, the following features are observed for the four modes: $r=6 \mu \mathrm{m}$. The mean value of strength oscillates for all the period of measurements, showing a slight increase from the highest values were found on August and September. $r=1.5 \mu \mathrm{m}$. The two strengths concerning these mode show the same behavior, while the second mode is always higher than the first one, two major events are here recognizable: The first begins on 14 March and suddenly ends in the afternoon of 20 March. For all the period around $1 \times 10^{-6} \mathrm{~cm}^{3}$ and the highest values were found on, September and October. $r$ $=0.25 \mu \mathrm{m}$. The strengths concerning these mode oscillates for all the period around $1 \times 10^{6} \mathrm{~cm}^{3}$ and the highest values were found on February and March. The strengths for the four modes radii have been also determined at several times during the day in order to detect a possible diurnal behavior.

\section{CONCLUSION}

On the basis of the present study the aerosol number concentration was determined indirectly, making use of the intensity of light scattered by particles. The scattered light intensity is proportional to the average number concentration of the aerosols. The easterly suspended particles carry fine particles and other pollutants mainly from the emission sources of Helwan ( $25 \mathrm{~km}$ from Cairo). Westerly winds stem from the Sahara, bring dry air masses, which in summer are warm and turbid because they are dust laden. The columnar volume radius distributions of aerosol 3-6 $\mu \mathrm{m}$ showed the aerosol optical depth is less than 0.31 for $\lambda$ $=500-630 \mathrm{~nm}$. The behavior in a more turbidity, when the aerosol optical depth is about 0.25 for $630-695 \mathrm{~nm}$ gives approximately fixed volume radius distributions of aerosol 5-8 $\mu \mathrm{m}$. The increase of the volume of particles with radius about $1-4 \mu \mathrm{m}$ is strongly related to the passage of desert in the period Khamassen (spring). The differences among the region is characterized mainly by their different climate change taken in consideration in the spectral region 250-900 nm. Most of the particles are greater than $10 \mu \mathrm{m}$ in aerodynamic diameter and $60-80 \%$ of particles are $5-10 \mu \mathrm{m}$ and are trapped in the nasopharyngeal region.

\section{REFFERENCES}

Barrett, J.C. and N.A. Webb, 1998. A comparison of some approximate methods for solving the aerosol general dynamic equation. J. Aerosol Sci., 29: 31-39.

Benkovitz, C.M., C.M. Berkowitz, R.C. Easter, S. Nemesure and R. Wagener et al., 1994. Sulfate over the Atlantic and adjacent continental regions: Evaluation of October and November 1986 using a three dimensional model driven by observationderived meteorology. J. Geophys. Res., 99: 725-756.

Chiemeka, I.U., 2006. Determination of aerosol mass, concentration, thickness of impaction and composition during the 1989/1990 harmattan season at Jos, Nigeria. JRPS, 2: 22-25. 
Chiemeka, I.U., M.O. Oleka and T.C. Chineke, 2007a. Determination of Aerosol Metal Composition and Concentration during the 2004/2005 Harmattan Season at Uturu, Nigeria. Adv. Sci. Techno., 1: 126-131.

Chiemeka, I.U., M.O. Oleka and T.C. Chineke, 2007b. Measurement of aerosol concentration variation by opto-electronic method. GJPAS, 13: 279-282.

Ferm, M., 1979. Method for determination of atmospheric ammonia. Atmosph. Environ., 13: 1385-1393.

McGraw, R., 1997. Description of aerosol dynamics by the quadrature method of moments. Aerosol Sci. Technol., 27: 255-265.

McGraw, R., P.I. Huang and S.E. Schwartz, 1995. Optical properties of atmospheric aerosols from moments of the particle size distribution. Geophys. Res. Lett., 22: 2929-2932.

McGraw, R., S. Nemesure and S.E. Schwartz, 1998. Properties and evolution of aerosols with size distributions having identical moments. J. Aerosol Sci., 29: 1-12.
Melnikova, I.N. and A.V. Vasilyev, 2005. Short-Wave Solar Radiation in the Earth's Atmosphere Calculation, Observation, Interpretation. SpringerVerlag, Berlin Heidelberg, Germany, pp: 303.

Peters, A., H. E. Wichmann, T. Tuch, J. Heinrich and J. Hyder, 1997. Respiratory effects are associated with the number of ultrafine particles. Am. J. Respir. Crit. Care Med., 155: 1376-83.

Utah, E.U., 1995. Aerosol optical density during the harmattan at Jos. Nigeria, Niger. J. Phys., 7: 67-71.

Van Dehulst, H.C., 1957. Light Scattering By Small Particles. John Willey and Sons, New York, pp: 52.

Weller, M., P. Plessing, H. Rentsch, J. Lattauschke and W. Hoyningen, 2000. Regional differences of column related aerosol parameters in Germany. Atmosph. Environ., 34: 5107-5118.

William, P.C. and W.S. Barbara, 2001. Environmental Science: A Global Concern. 6th Edn., McGrawHill Company New York, pp: 404-415. 